\title{
Índice de libros reseñados y recensionados
}

Aranda Doncel, J. El Cristo de la Expiración y la Semana Santa de Córdoba. Cuatro siglos de historia (1612-2018). Córdoba: Cofradía del Cristo de la Expiración, 2019, 404 pp. [978-84-12-06984-6]

Arias Montano, B. Apología de la Biblia Regia. Edición a cargo de Antonio Dávila Pérez. Alcañiz-Lisboa: CEC, 2019, 489 pp. [978-84-179-9905-6]

Asti, F. - Salato, N. - Cibelli, E. (a cura di), La misericordia: Forma relationis. Prospettive ermeneutiche, Napoli: Pontificia Facoltà Teologica dell'Italia meridionale. Sezione S. Tommaso d'Aquino, 2019, 262 pp.

Bady, G. y Cuny, D. (eds.), Les polémiques religieuses $d u I^{e r}$ au IVème siècle de nôtre ère, París: Beauchesne, 2019, 450pp. [978-2-7010-2277-2]

Bassi, S. (a cura di), Costellazioni concettuali tra Cinquecento e Settecento. Filosofia, religione, política, Firenze: Leo S. Olschki Editore, 2019, VIII+187 pp. [978-88-222-6633-0]

Bejar Baca, J. S., Los milagros de Jesús. Una visión integradora, Barcelona: Herder, 2018.

Benedetto XVI (in dialogo con il rabbino Arie Folger), Ebrei e Cristiani, Città del Vaticano - Cinisello Balsamo (Milano): Librería Editrice Vaticana - EdizioniSan Paolo, 2019, 142 pp. [978-88-922-1892-5]

Berdugo Villena, T., Identidad del Concilio de Elvira. Granada: Universidad, 2019, $377 \mathrm{pp}$.

Bourgine, B. Bible oblige. Essai de théologie biblique. París: Ed. Cerf, 2019, 303 pp. [978-2-204-13438-5]

Camaioni, M., Il Vangelo e l'Anticristo. Bernardino Ochino tra francescanesimo ed eresia (1487-1547), Napoli: Società Editrice Il Mulino, 2018, XXXI+604 pp. [978-88-15-27853-1]

Cancedo García, J. L., Algunas consideraciones sobre el pensamiento de Martin Heidegger. Señales de su trasfondo religioso, Burgos: Fonte Monte Carmelo, 2019, 211 pp.

Capizzi, A Platón en su tiempo. La infancia de la filosofía y sus pedagogos, Zaragoza: Prensas de la Universidad de Zaragoza, 2019, 287 pp. [978-84-17633-89-9]

Cárcel Ortiz, V., Diario de Federico Tedeschini (1931-1939). Nuncio y cardenal entre la Segunda República y la Guerra Civil española, Barcelona: Balmes, 2019, 1086 pp. [978-84-210-0697-9]

Cheryl Exum, J., Art as Biblical Commentary. Visual Criticism from Hagar the Wife of Abraham to Mary the Mother of Jesus. London: T\&TClark, 2019. 270 pp.

Coello de la Rosa, A., Gathering Souls: Jesuit Missions and Missionaries in Oceania (1668-1945), Leiden-Boston: Brill, 2019, 115 pp. [978-90-04-39485-8] 
Egido, A., El diálogo de las lenguas y Miguel de Cervantes. Zaragoza: Prensas de la Universidad de Zaragoza, 2019, 141 pp. [978-84-1787-341-7]

Escrivá de Balaguer, J., Amigos de Dios. Homilias, Edición crítico-histórica preparada por Antonio Aranda, Madrid: Ediciones Rialp, 2019, LII + 955 pp. [978-84-321-5105-7]

Fabbri, L., Mater florum. Flora e il suo culto a Roma, Firenze: Leo S. Olschi Editore, 2019, XIII+278 pp. [978-88-222-6619-4]

Florensa Parés, J., Josep Calassanç i Gastó, mestre d'escola, Barcelona: Escola Pia de Catalunya, 2017, 314 pp. [978-84-697-7452-6]

Fürst, F. - Hengstermann, Ch. (hgs.), Ralph Cudworth. Predigt vor dem Unterhaus und andere Schriften, Münster: Aschendorff Verlag, 2018, 311 pp. [978-3-402-13726-0]

García Bazán, F., La niñez y adolescencia del General San Martín en Málaga, Buenos Aires, 2018, 24 pp.

Hall-Van den Elsen, C. Fuerza e intimismo: Luisa Roldán, escultora (1652-1706). Madrid: Consejo Superior de Investigaciones Científicas, 2018, 352 pp. [978-84-00-10377-4]

Insausti, G., Verdad y belleza: la pasión de Gerard Manley Hopkins, Pamplona: Ediciones Universidad de Navarra, 2019, 156 pp. [978-84-313-3370-6]

Karl, K. - Uhrig, C. (eds.), Zeit und Geist, Münster: Aschendorff, 2017, 134 pp. [978-3-402-13249-4]

La Bella, G. Los jesuitas. Del Vaticano II al papa Francisco. Bilbao: Mensajero, 2019, 459 pp. [978-84-27143-91-3]

Lallemant, L. (Salin, D., nueva edición, García Rodríguez, J. A., edición revisada, y Marteles López, J. L, Sánchez Del Río, L. T., Serón Puertas, E. y Tornos Cubillos, A., traducción), Doctrina espiritual [París 2011], Bilbao - Santander - Madrid: Mensajero - Sal Terrae - Universidad Pontificia Comillas, 2017, 479 pp. [978-84-8468-705-4 y 978-84-271-4124-7].

Leclerc du Sablon, J., Vivir al estilo de Jesús. Itinerario de una vida en misión, Madrid: Narcea de Ediciones, 2019, 155 pp. [978-84-277-2564-5]

Martínez de Bujanda, J., Censura de la Inquisición y de la Iglesia en España (1520-1966), Madrid: Biblioteca de Autores Cristianos, 2019, XVII+274 pp. [978-84-220-2086-8]

Massaro, Th., Mercy in action. The Social Teachings of Pope Francis, Lanham (Maryland, EE. UU.): Rowman \& Littlefield, 2018, 208 pp [978-1-4422-7174-6]

Muñoz Rojo, M., El hombre para la historia. El cardenal Portocarrero (1635-1709). Córdoba: Editorial Universidad de Córdoba, 2019, 384 pp. [978-84-9927-437-9] 
Nixey, C., La edad de la penumbra. Cómo el cristianismo destruyó el mundo clásico, Madrid: Taurus, 2018, 318 pp. + mapa + ilustraciones $\mathrm{b} / \mathrm{n}$ y color [978-84-306-1954-2]

Orique, D. TH. - Roldan-Figueroa, R. (eds.), Bartolomé de las Casas, O.P. History, Philosophy, and Theology in the Age of European Expansion, Leiden-Boston: Brill, 2019, XX+485 pp. [978-90-04-36973-3]

Panikkar, R., Iniciació als Veda, Barcelona: Fragmenta Editorial, 2018, 98 pp. [978-8415518-96-9]

Parker, G., Carlos V. Una nueva vida del emperador, Barcelona: Editorial Planeta, 2019, 990 pp. [978-84-08-20477-0]

Pérez Chico, D., Wittgenstein y el escepticismo. Certeza, paradoja y locu$r a$, Zaragoza: Prensas de la Universidad de Zaragoza, 2019, 347 pp. [978-84-17633-39-4]

Pérez-Mallaína Bueno, P. E., Las Atarazanas de Sevilla. Ocho siglos de historia del arsenal del Guadalquivir, Sevilla: Editorial Universidad de Sevilla - Instituto de Cultura y de las Artes de Sevilla - Ayuntamiento de Sevilla - Diputación de Sevilla, 2019, 773 pp. [978-84-472-1977-3]

Pittel, S., Geschichtliche Realität und Kreuz, Regensburgo: Verlag Friedrich Pustet, 2018, 432 pp. [978-3-7917-3006-6]

Puglisi, G. - Montinaro, G. (a cura di), Martin Lutero cinquecento anni dopo, Firenze: Casa Editrice Leo S. Olschki, 2019, VI+130 pp. [978-88-222-6636-1]

Questier, M., Dynastic Politics and the British Reformations, 15581630, Oxford: Oxford University Press, 2019, XV+499 pp. [978-0-19-882633-0]

Quintana Trías, Ll., Art i blasfèmia. El cas Veronese, Barcelona: Fragmenta Editorial, 2019, 125 pp. [978-84-15518-95-2]

Quintana Trías, Ll., Arte y blasfemia. El caso Veronese. Barcelona: Fragmenta, 2019, 140 pp. [978-84-17796-15-0]

Raguer i Suñer, H., Tres catalans de la tercera Espanya. Carrasco Formiguera, Domènec Batet, Vidal i Barraquer, Barcelona: Abadia de Montserrat (Biblioteca Serra d'Or no 505), 2018, 185 pp. [978-84-9191-026-8]

Raguer i Suñer, H., Escrits dispersos d'història, Barcelona: Institut d'Estudis Catalans - Diputació de Barcelona, 2018, 574 pp. [978-849965-417-1] y [978-84-9803-842-2]

Rebolo González, L. J., Biografia teológica de la transición política española (1965-1982), Boadilla del Monte (Madrid): PPC, 2018, 166 pp. [978-84-288-3304-2]

Rotundo, E., La kenosi di Unus de Trinitate. Cristo dal Padre nello Spirito: come é in Cielo così in terra. Una proposta di cristologia kenotica, Assisi: Cittadella Editrice, 2017, 460 pp. 
Saranyana, J.-I., La teología cristiana a la modernitat. De l'albada del segle XVI al llindar de la Il-lustració, Valls: Cossetània Edicions, 2019, 188 pp. [978-84-9034-819-2]

Sarmiento, A. - Zorroza, M ${ }^{\mathrm{a}}$. I. (eds.), Francisco de Vitoria, F., Comentarios a la Prima secundae de la Summa Theologiae de Santo Tomás, Vol IV. De donis, beatitudinibus, fructibus, vitiis et peccatis. Sobre los dones, las bienaventuranzas, los frutos, vicios y pecados, Pamplona: Ediciones Universidad de Navarra, 2019, 481 pp. [978-84-313-3340-9]

Schlotheuber, E. - Liewert, A., Musik aus Paradiese. Die mittelalterlichen handschriften der Dominikanerinnen aus Paradiese bei Soest, Münster: Aschendorff Verlag, 2019, 40 pp. [978-3-402-24615-3]

Secchi, P., Studi cusaniani, Firenze: Leo S. Olschki Editore, 2018, X+179 pp. [978-88-222-6569-2]

Silvano, M. D. (ed.), Bibliografía mariana, Roma: Edizioni Marianum, 2019, 491 pp.

Silvestrini, F. (a cura di), La memoria del chiostro. Studi di storia e cultura monástica in ricordo di Padre Pierdamiano Spotorno O.S.B. archivista, bibliotecario e storico de Vllombrosa (1936-2015), Firenze: Leo S. Olschki Editore, 2019, X+767 pp. [978-88-222-6590-6]

Somavilla Rodríguez, E. (dir.), El transhumanismo en la sociedad actual, Guadarrama (Madrid): Editorial Agustiniana, 2019, 279 pp. [978-84-92645-67-1]

Tur Palau, V., Teología y creatividad, Valencia: Facultad de Teología San Vicente Ferrer, 2018, 469 pp.

Vannier, M.-A. (dir.), Les chemins spirituels dans la Mystique Rhénane et la Devotio Moderna, París: Beauchesne Éditeur, 2018, 283 pp. [978-2-7010-2278-9]

Vannier, M.-A. Maître Eckhart Prédicateur, París: Beauchesne, 2018, 858 pp. [978-2-7010-2287-1] 